Journal of Mathematics and Statistics Studies

ISSN: 2709-4200

DOI: $10.32996 /$ jmss

Journal Homepage: www.al-kindipublisher.com/index.php/jmss

\title{
Evaluating Percentage Rotatability For The Small Box - Behnken Design
}

\author{
Lawrence Chizoba Kiwu ${ }^{1}$ (D) $\triangle$, Desmond Chekwube Bartholomew ${ }^{2}$ (D), Fidelia Chinenye Kiwu- \\ Lawrence $^{3}$ \& (D), Chukwudi Paul Obite 4 (D) and Okafor Ikechukwu Boniface ${ }^{5}$ (D) \\ ${ }^{124}$ Department of Statistics, Federal University of Technology, Owerri, Nigeria \\ ${ }^{3}$ Department of Statistics, Abia State University Uturu, Abia State, Nigeria \\ ${ }^{5}$ Department of Statistics, Convenant Polytechnic Aba, Nigeria \\ $\triangle$ Corresponding Author: Lawrence Chizoba Kiwu, E-mail: Lawrence.kiwu@futo.edu.ng
}

\section{ARTICLE INFORMATION}

Received: June 08, 2021

Accepted: August 02, 2021

Volume: 2

Issue: 2

DOI: 10.32996/jmss.2021.2.2.3

\section{KEYWORDS}

Response Surface Methodology,

Rotatability,Small Box-Behnken

Design and Percentage

\section{ABSTRACT}

Rotatability property for the Small Box-Behnken design is discussed in this paper. This paper aimed at applying a measure of obtaining percentage rotatability on the Small Box-Behnken designs to determine if the Small Box-behnken designs are rotatable or not and investigated the extent of rotatability in terms of percentage. The factors, $q$, considered range from 3 to 11. The results showed that for factors $q$, the Small BoxBehnken design is rotatable for $q=3$ factors, near rotatable for $q=4,7$ factors and not rotatable for $q=5,6,8,9,10$ and 11 factors.

\section{Introduction}

Considering the importance of experimental design and modelling of experiments, the Response Surface Methodology (RSM) is a combination of mathematical and statistical procedures that is helpful in the modeling and analysis of problems with the aim of optimizing the response of interest, Montgomery (2005). RSM procedure is basically presented using two important models, the first-degree model of order, $r,(r=1)$, given in (1),

$$
y=\tau_{0}+\sum_{j=1}^{q} \tau_{j} x_{j}+\varepsilon_{j i}
$$

and the second degree modelof order, $r,(r=2)$, given in (2),

$$
y=\tau_{0}+\sum_{j=1}^{q} \tau_{j} x_{j}+\sum_{j<i} \tau_{j i} x_{j} x_{i}+\sum_{j=1}^{q} \tau_{j j} x_{j}^{2}+\varepsilon_{j i}
$$

Kiwu-Lawrence et al. (2021), in their work, used the second-order response surface model to evaluate and compare the performances of three classes of Central Composite Design: Central Composite Circumscribed Design (CCCD), Central Composite Inscribed Design (CCID) and Central Composite Face-Centered Design (CCFD) using the A-, D-, and G-efficiencies for factors, k, that ranges from 3 to 10, with 0-5 centre points. It was shown from their result that the CCDs (CCCD, CCFD and CCID) are at their best when the G-efficiency is employed for all the factors considered while the CCID, in particular, behaves poorly when using the A- and D-efficiencies. Chigbu and Ohaegbulem (2011), in their study, applied the D-optimality criterion, which is a response

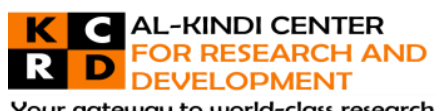

Your gateway to world-class research

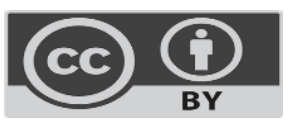

Published by Al-Kindi Center for Research and Development. Copyright (c) the author(s). This open access article is distributed under a Creative Commons Attribution (CC-BY) 4.0 license 
surface design optimality criteria to compare partially replicated cube and star portions of the rotatable and orthogonal CCD. They concluded that when the cube portion is replicated, the D-optimal performance of the CCD is enhanced better than replicating the star portion.

Designs that are based on three-level incomplete factorial designs for effectively fitting response surface model given in (2) were proposed by Box and Behnken (1960). The proposed designs gained popularity because they effectively obtain the maximum amount of information in a short period of time and their wide applications in industrial processes: see, for example, Aslan and Cebeci (2006). The designs proposed by Box and Behnken (1960), are rotatable or near rotatable. Rotatabilty is a property that ensures that from points equidistant from the design centre, the variance of a predicted response at a point remains constant at all such points, Mutai et al. (2013). Given that there is no advanced knowledge on how the response surface will orient itself with respect to its axes, it becomes necessary to adopt the rotatability property to explore the study of response surface design, Onukogu and Chigbu (2002). Since the introduction of the method of assessing rotatability or near-rotatability by Box and Hunter (1957), several efforts have been made at obtaining the rotatability or near-rotatability of the second-order design over a particular region of interest.

In other to obtain design rotatability or near-rotatability, researchers have developed different measures of design rotatability; the most common measures are given by Ekman (1994). In his study, graphical techniques were used to explore the variance function and the study concluded that it is not possible to construct a design with equal variance over the design space. Draper and Pukelsheim (1990), suggested a criterion for rotatability, which is invariant under design rotation. The criterion was provided for central composite designs and it was concluded that the criterion could be extended to higher-degree models. Draper and Guttman (1988) suggested a measure of rotatability for design space, but the measure has the limitations of not providing the extent of design rotatability.

Khuri (1988), introduced a measure that quantifies rotatability in percentage. That is, presenting the extent of rotatability for a design space in terms of percentage. The proposed measures have some desirable advantages. Hence, in this study, the proposed measure of rotatability by Khuri (1988) was used to ascertain the extent of rotatability in terms of percentage for the Small Box-Behnken designs. The Small Box-Behnken designs which fits the second-order response surface model with only a much smaller run size and with reasonably high D-efficiencies was developed by Zhang et al. (2011) as an alternative to the BoxBehnken design, since the Box Behnken designs have the limitation of a rapid increase in design run size as the number of factors, $k$, increases. The Orthogonality property of the original Box-Behnken design is preserved in the Small Box-Behnken designs, as pointed by Zhang et al. (2011). However, their work was silent on whether the rotatability property of the BoxBehnken designs is preserved in the Small Box-Behnken designs or not and that form the basis of this study. In this study, our aim is to evaluate the rotatability property of the Small Box-Behnken design in terms of percentage and recommend the design that is rotatable or not based on the percentage.

\section{Methods and Materials}

\subsection{Condition for Rotatability}

Rotatability is characterized by the information matrix, $X^{\prime} X$. where, $X^{\prime} X$, is called esign moments. In general, a design moment for a model such as equation (2) of order $r$ and in $q$ input variables is denoted by $\left[1^{\delta 1}, 2^{\delta 2}, \ldots, q^{\delta q}\right]$ and is equal to equation (3), where $\delta_{i}, i=1, \ldots, q$, are non-negative numbers and $x_{u i}$ represents the level for the ithfactor used in the $u^{\text {th }}$ experimental runs, $(i=1,2, \ldots, q ; u=1,2, \ldots, n)$. Given that the design matrix, $(X)$, for $q$ factors is represented by $x_{1}, x_{2}, \ldots, x_{q}$ as an $N \times q$ matrix whose $u_{t h}$ row; $x_{u}^{\prime}=\left(x_{u 1}, x_{u 2}, \ldots, x_{u q}\right)$ represents the coded factor levels to be which is be used in the $u_{t h}$ row of the design space as in (2). $\left[1^{\delta 1}, 2^{\delta 2}, \ldots, q^{\delta q}\right]$ is used to denote the general design moment of (2) where;

$\left[1^{\delta 1}, 2^{\delta 2}, \ldots, q^{\delta q}\right]=\sum_{u=1}^{n} X_{u i}^{\delta 1}, X_{u 2}^{\delta 2}, \ldots, q_{u q}^{\delta q}$

According to [2], for model such as (2) the necessary and sufficient condition for fitting such model of order $r$ and in $q$ factor so as to achieve rotatablility is that the general design moment of order $\delta(\delta=0,1,2, \ldots, 2 r)$ must be of the form; $\left[1^{\delta 1}, 2^{\delta 2}, \ldots, k^{\delta q}\right]$

where, 
$\left\lfloor 1^{\delta_{1}}, 2^{\delta_{2}}, \ldots, k^{\delta_{k}}\right\rfloor=0$; if any $\delta_{i}$ is odd

$=\frac{\theta_{\delta} \prod_{i=1}^{k} \delta_{i} !}{2^{\delta / 2} \prod_{i=1}^{k}\left(\delta_{i} / 2\right) !}$; if any $\delta_{\mathrm{i}}$ are even

Where, $\theta_{\delta}$, depends on $r, \delta$ and $n$; Box and Hunter (1957).

\section{Second-Order Requirement for Rotatability}

The general requirement for (2) rotatable is that the even moments must have the following form:

$\left[j^{2}\right]=\frac{1}{N} \sum_{u=1}^{N} x_{u j}^{2}=\lambda_{2}$

$\left[j^{2} i^{2}\right]=\frac{1}{N} \sum_{u i}^{N} x_{u j}^{2} x_{u i}^{2}=\lambda_{4}$

$\left[j^{4}\right]=\frac{1}{N} \sum_{u=1}^{N} x_{u j}^{4}=3 \lambda_{4}\left(j^{4}\right)=3 \lambda_{4}$

where, $j=(1,2, \ldots, q), j=(1,2, \ldots, q),(j \neq i), q$ is the number of factors, $\lambda_{2}$ and $\lambda_{4}$ are the second-order of the design moments and fourth-order of the design moments, respectively, for any second-order design. Here, the odd moments of the order less than or equal to four is set to zero. Adding centre points to the design matrix $X$, does not change the general form of the design moments once design moments of $X$, satisfies the rotatability condition, Box and Behnken (1959).

\section{Rotatability property}

In many cases, the scaled prediction variance $N\left(\frac{\operatorname{var}[(X)]}{\delta^{2}}\right)$ of a response surface design is the general property in experimental design used to show how the design points are distributed within the region of interest. The distribution of design points in the experimental region is characterized by the design moments and the design moment is a function of the model being fitted. The design moment comes from the moment matrix, $M$, given by

$M=\frac{X^{\prime} X}{N}$, where, $\left(X^{\prime} X\right)$, is referred to as the information matrix and $N$ is number of runs.

For a second-degree model, where $q=3$, the moment matrix is given by;

$M=\left[\begin{array}{cccccccccc}x_{0} & x_{1} & x_{2} & x_{3} & x_{1} x_{2} & x_{1} x_{3} & x_{2} x_{3} & x_{1}^{2} & x_{2}^{2} & x_{3}^{2} \\ 1 & 0 & 0 & 0 & 0 & 0 & 0 & \sigma_{2} & \sigma_{2} & \sigma_{2} \\ 0 & \sigma_{2} & 0 & 0 & 0 & 0 & 0 & 0 & 0 & 0 \\ 0 & 0 & \sigma_{2} & 0 & 0 & 0 & 0 & 0 & 0 & 0 \\ 0 & 0 & 0 & \sigma_{2} & 0 & 0 & 0 & 0 & 0 & 0 \\ 0 & 0 & 0 & 0 & \sigma_{22} & 0 & 0 & 0 & 0 & 0 \\ 0 & 0 & 0 & 0 & 0 & \sigma_{22} & 0 & 0 & 0 & 0 \\ 0 & 0 & 0 & 0 & 0 & 0 & \sigma_{22} & 0 & 0 & 0 \\ \sigma_{2} & 0 & 0 & 0 & 0 & 0 & 0 & \sigma_{4} & \sigma_{22} & \sigma_{22} \\ \sigma_{2} & 0 & 0 & 0 & 0 & 0 & 0 & \sigma_{22} & \sigma_{4} & \sigma_{22} \\ \sigma_{2} & 0 & 0 & 0 & 0 & 0 & 0 & \sigma_{22} & \sigma_{22} & \sigma_{4}\end{array}\right]$

(see Borkowski 2003), 


\section{Rotatability Measure}

According to Montgomery (2005), the model in (2) of order $r$, with $q$ factors $\left(x_{1}, x_{2}, \ldots, x_{q}\right)$. If the factors are codes as in (6) and the model is fitted in a spherical region, we have the following representation, $\sum_{u=1}^{n} Z_{u j}=0 ; \quad j=1,2, \ldots, q$

$$
\sum_{u=1}^{n} Z_{u j}^{2}=\lambda_{2} ; \quad j=1,2, \ldots, q
$$

So that, $Z_{u j}$ represents the coded value of $\boldsymbol{X}_{u j}$, and $\lambda_{2}$ is some positive constant. By adapting the notion described in equation (4) to the coding variables (factors), the equalities described in equation (6) is hence, in the form of (7);

$\sum_{u=1}^{n} Z_{u j}=(j)=0 ; \quad j=1,2, \ldots, q$

$\sum_{u=1}^{n} Z_{u j}^{2}=\left(j^{2}\right)=\lambda_{2} ; \quad j=1,2, \ldots, q$

\section{Rotatable Design and the Canonical Representation of the information matrix ( $\left.Z^{\prime} Z\right)$}

Let $Z_{r}$ denote the $Z$ matrix when the design is rotatable. In this case, the vector $\quad v\left(Z_{r}^{\prime} Z_{r}\right)$ is given by

$v\left(Z^{\prime} Z\right)=\theta_{0} w_{0}+\theta_{2} w_{2}+\ldots+\theta_{2 d} w_{2 d}$

where, $w_{\delta}(\delta=0,2, \ldots, 2 r)$, represents a vector, with dimension, $h^{*} \times 1, h^{*}=\frac{h(h+1)}{2}$ and $h$, is the number of parameters in (2). The quantity in (9) represents scale-free quantities;

$A v\left(Z^{\prime} Z\right)=u\left(Z^{\prime} Z\right)$

Where, the elements of, $A=1 / \gamma^{\delta}$, corresponds one-to-one with the elements of $v\left(Z^{\prime} Z\right)$ and the elements of $A$ corresponds to design moments of order, $\delta ;(10)$ shows the canonical expression for $\mathrm{u}\left(\mathrm{Z}^{\prime} Z\right)$ and it is obtained from (12) and (8)

$$
\begin{gathered}
\mathrm{u}\left(\mathbf{Z}^{\prime} \mathbf{Z}\right)=\frac{\sum_{m=0}^{r} \theta_{2 m} w_{2 m}}{\gamma^{2 m}} \\
=n w_{0}+w_{2}+\sum_{m=2}^{r} q_{2 m} w_{2 m}
\end{gathered}
$$

where,

$q_{2 m}=\frac{\theta_{2 m}}{\gamma^{2 m}}, \quad m=2,3, \ldots, r$

Supposed that the $N$-point design, $X$, for the fitting model in the (1) and (2), with the input variables (factors), coded as in equation (7). The corresponding vector, $u\left(Z^{\prime} Z\right)$, in equation (9) is given by

$\mathrm{u}\left(\mathrm{Z}^{\prime} \mathrm{Z}\right)=n w_{0}+w_{2}+\mathrm{u}^{*}(\mathrm{Z} \mathrm{Z})$

where, $w_{0}$ and $w_{2}$ are the same as in equation (10), and for a design with an equal number of runs, $\mathrm{n},(12)$ holds for a rotatable design.Except for those that correspond to design moments of order, $\delta=0$, and even design moments of order, $\delta=2$, which are equal to 0 , the elements of $u^{*}\left(Z^{\prime} Z\right)$ are equal to the corresponding elements of $u(Z Z)$. The percentage rotatability measure is represented in (13), Khuri (1988). 
$\phi_{n}(X)=100 \times\left\{\sum_{m=2}^{r}\left[\mathrm{u}^{*}\left(\mathrm{Z}^{\prime} \mathrm{Z}\right) \mathrm{w}_{2 \mathrm{~m}}\right]^{2} /\left\|w_{2 m}\right\|^{2}\right\} /\left\|\mathrm{u} *\left(\mathrm{Z}^{\prime} \mathrm{Z}\right)\right\|^{2}$

7. Evaluating the percentage rotatability for the Small Box-Behnken Design

The expanded design matrix for Small Box-Behnken design for $q=3$ is given by

$Z=\left[\begin{array}{cccccccccc}X_{0} & X_{1} & X_{2} & X_{3} & X_{1} X_{2} & X_{1} X_{3} & X_{2} X_{3} & X_{1}^{2} & X_{2}^{2} & X_{3}^{2} \\ 1 & -1 & -1 & 0 & 1 & 0 & 0 & 1 & 1 & 0 \\ 1 & 1 & -1 & 0 & -1 & 0 & 0 & 1 & 1 & 0 \\ 1 & -1 & 1 & 0 & -1 & 0 & 0 & 1 & 1 & 0 \\ 1 & 1 & 1 & 0 & 1 & 0 & 0 & 1 & 1 & 0 \\ 1 & -1 & 0 & -1 & 0 & 1 & 0 & 1 & 0 & 1 \\ 1 & 1 & 0 & -1 & 0 & -1 & 0 & 1 & 0 & 1 \\ 1 & -1 & 0 & 1 & 0 & -1 & 0 & 1 & 0 & 1 \\ 1 & 1 & 0 & 1 & 0 & 1 & 0 & 1 & 0 & 1 \\ 1 & 0 & -1 & -1 & 0 & 0 & 1 & 0 & 1 & 1 \\ 1 & 0 & 1 & -1 & 0 & 0 & -1 & 0 & 1 & 1 \\ 1 & 0 & -1 & 1 & 0 & 0 & -1 & 0 & 1 & 1 \\ 1 & 0 & 1 & 1 & 0 & 0 & 1 & 0 & 1 & 1\end{array}\right]$.

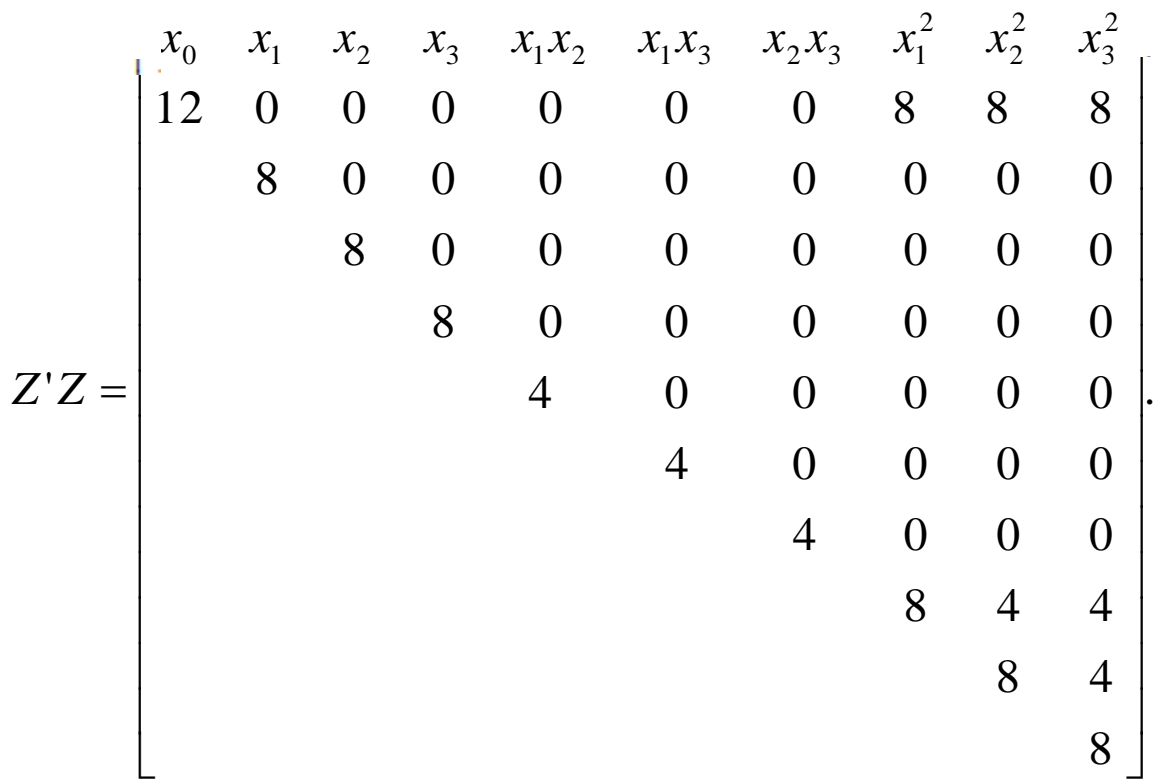

For the average of all even design moments of order $2, \gamma^{2}$ is given as;

$$
\gamma=\left[\sum_{j=1}^{q} j^{2} / q\right]^{1 / 2}
$$


Where, $j^{2}=\sum_{j=1}^{\mathrm{q}} \mathrm{x}_{j}^{2}=8$.

Therefore, $\gamma=\left[\frac{8+8+8}{3}\right]^{1 / 2}=8^{1 / 2}$.

$A=\gamma^{\delta}$

The following steps from 1 to 5 were used to calculate the values of $\mathrm{w}_{0}, \mathrm{w}_{2}$, and $\mathrm{w}_{4}$ entries in Table 1 :

1. $\mathrm{W}_{0}, \mathrm{w}_{2}, \mathrm{w}_{4}$, can be obtained from equation (8) through equation (11), given that; $\mathrm{u}\left(\mathrm{Z}^{\prime} \mathrm{Z}\right)=\frac{\sum_{m=0}^{r} \theta_{2 m} w_{2 m}}{\tau^{2 m}}$, we shall use this notation to obtain the values of $\mathrm{W}_{0}, \mathrm{w}_{2}, \mathrm{w}_{4}$, for $m=0,1,2$.

2. If $m=0$, we have that $\mathrm{u}\left(\mathrm{Z}_{\mathrm{r}}{ }^{\prime} \mathrm{Z}_{\mathrm{r}}\right)=\frac{\theta_{0} w_{0}}{\gamma^{0}}$; recall that, from equation (8), the elements of $w_{0}=1$ for only the first entry, all other entries are 0 . From (4), it can be seen that $\theta_{0}=n$. Since for $q=3, n=12, u\left(Z^{\prime} Z\right)=12$, therefore, $\mathrm{w}_{\mathrm{o}}=1$.

3. $w_{2 m}=0$, except for those elements that corresponding to even design moments of order $2 \mathrm{~m}$. Also note that, $\theta_{2}=$ $\lambda_{2}$ by the coding scheme of equation (7). Hence, by equation (11), $\theta_{2}=\gamma^{2}$.

If $m=1$, we have that $w_{2}=\frac{\gamma^{2}\left(\mathrm{u}\left(\mathrm{Z}^{\prime} \mathrm{Z}\right)\right)}{\theta_{2}}=1$; that is, $\theta_{2}=\tau^{2}=8$ and $\mathrm{u}\left(\mathrm{Z}^{\prime} \mathrm{Z}\right)=1$.

4. If $m=2$, we have a case of the second pure moment and second mixed moment.

5. For the second pure moment, we have that; $\mathrm{w}_{4}=\frac{2\left[(\mathrm{u}(\mathrm{Z} Z)) \gamma^{4}\right]}{\theta_{4}}$, and for the second mixed moment, we have; $\mathrm{w}_{4}=\frac{\left[\left(\mathrm{u}\left(\mathrm{Z}^{\prime} \mathrm{Z}\right)\right) \gamma^{4}\right]}{\theta_{4}}$.

Table 1: Computation of percentage rotatability for factor $\mathbf{k}=\mathbf{3}$.

\begin{tabular}{|l|l|l|l|l|l|l|l|l|}
\hline$\delta$ & $\mathrm{v}\left(\mathrm{Z}^{\prime} \mathrm{Z}\right)$ & $\delta / 2$ & $\mathrm{~A}$ & $\mathrm{u}\left(\mathrm{Z}^{\prime} \mathrm{Z}\right)$ & $\mathrm{u} *\left(Z^{\prime} Z\right)$ & $\mathrm{w}_{0}$ & $\mathrm{w}_{2}$ & $\mathrm{w}_{4}$ \\
\hline $\mathbf{0}$ & 12 & 0 & 1 & 12 & 0 & 1 & 0 & 0 \\
\hline $\mathbf{1}$ & 0 & 0.5 & 0.3535534 & 0 & 0 & 0 & 0 & 0 \\
\hline $\mathbf{1}$ & 0 & 0.5 & 0.3535534 & 0 & 0 & 0 & 0 & 0 \\
\hline $\mathbf{1}$ & 0 & 0.5 & 0.3535534 & 0 & 0 & 0 & 0 & 0 \\
\hline $\mathbf{2}$ & 0 & 1 & 0.125 & 0 & 0 & 0 & 0 & 0 \\
\hline $\mathbf{2}$ & 0 & 1 & 0.125 & 0 & 0 & 0 & 0 & 0 \\
\hline $\mathbf{2}$ & 0 & 1 & 0.125 & 0 & 0 & 0 & 0 & 0 \\
\hline $\mathbf{2}$ & 8 & 1 & 0.125 & 1 & 0 & 0 & 1 & 0 \\
\hline $\mathbf{2}$ & 8 & 1 & 0.125 & 1 & 0 & 0 & 1 & 0 \\
\hline
\end{tabular}




\begin{tabular}{|c|c|c|c|c|c|c|c|c|}
\hline 2 & 8 & 1 & 0.125 & 1 & 0 & 0 & 1 & 0 \\
\hline 2 & 8 & 1 & 0.125 & 1 & 0 & 0 & 1 & 0 \\
\hline 2 & 0 & 1 & 0.125 & 0 & 0 & 0 & 0 & 0 \\
\hline 2 & 0 & 1 & 0.125 & 0 & 0 & 0 & 0 & 0 \\
\hline 3 & 0 & 1.5 & 0.0441942 & 0 & 0 & 0 & 0 & 0 \\
\hline 3 & 0 & 1.5 & 0.0441942 & 0 & 0 & 0 & 0 & 0 \\
\hline 3 & 0 & 1.5 & 0.0441942 & 0 & 0 & 0 & 0 & 0 \\
\hline 3 & 0 & 1.5 & 0.0441942 & 0 & 0 & 0 & 0 & 0 \\
\hline 3 & 0 & 1.5 & 0.0441942 & 0 & 0 & 0 & 0 & 0 \\
\hline 3 & 0 & 1.5 & 0.0441942 & 0 & 0 & 0 & 0 & 0 \\
\hline 2 & 8 & 1 & 0.125 & 1 & 0 & 0 & 1 & 0 \\
\hline 2 & 0 & 1 & 0.125 & 0 & 0 & 0 & 0 & 0 \\
\hline 3 & 0 & 1.5 & 0.0441942 & 0 & 0 & 0 & 0 & 0 \\
\hline 3 & 0 & 1.5 & 0.0441942 & 0 & 0 & 0 & 0 & 0 \\
\hline 3 & 0 & 1.5 & 0.0441942 & 0 & 0 & 0 & 0 & 0 \\
\hline 3 & 0 & 1.5 & 0.0441942 & 0 & 0 & 0 & 0 & 0 \\
\hline 3 & 0 & 1.5 & 0.0441942 & 0 & 0 & 0 & 0 & 0 \\
\hline 3 & 0 & 1.5 & 0.0441942 & 0 & 0 & 0 & 0 & 0 \\
\hline 2 & 8 & 1 & 0.125 & 1 & 0 & 0 & 1 & 0 \\
\hline 3 & 0 & 1.5 & 0.0441942 & 0 & 0 & 0 & 0 & 0 \\
\hline 3 & 0 & 1.5 & 0.0441942 & 0 & 0 & 0 & 0 & 0 \\
\hline 3 & 0 & 1.5 & 0.0441942 & 0 & 0 & 0 & 0 & 0 \\
\hline 3 & 0 & 1.5 & 0.0441942 & 0 & 0 & 0 & 0 & 0 \\
\hline 3 & 0 & 1.5 & 0.0441942 & 0 & 0 & 0 & 0 & 0 \\
\hline 3 & 0 & 1.5 & 0.0441942 & 0 & 0 & 0 & 0 & 0 \\
\hline 4 & 4 & 2 & 0.015625 & 0.0625 & 0.0625 & 0 & 0 & 1 \\
\hline 4 & 0 & 2 & 0.015625 & 0 & 0 & 0 & 0 & 0 \\
\hline 4 & 0 & 2 & 0.015625 & 0 & 0 & 0 & 0 & 0 \\
\hline 4 & 0 & 2 & 0.015625 & 0 & 0 & 0 & 0 & 0 \\
\hline 4 & 0 & 2 & 0.015625 & 0 & 0 & 0 & 0 & 0 \\
\hline 4 & 0 & 2 & 0.015625 & 0 & 0 & 0 & 0 & 0 \\
\hline 4 & 4 & 2 & 0.015625 & 0.0625 & 0.0625 & 0 & 0 & 1 \\
\hline 4 & 0 & 2 & 0.015625 & 0 & 0 & 0 & 0 & 0 \\
\hline 4 & 0 & 2 & 0.015625 & 0 & 0 & 0 & 0 & 0 \\
\hline
\end{tabular}




\begin{tabular}{|l|l|l|l|l|l|l|l|l|}
$\mathbf{4}$ & 0 & 2 & 0.015625 & 0 & 0 & 0 & 0 & 0 \\
\hline $\mathbf{4}$ & 0 & 2 & 0.015625 & 0 & 0 & 0 & 0 & 0 \\
\hline $\mathbf{4}$ & 4 & 2 & 0.015625 & 0.0625 & 0.0625 & 0 & 0 & 1 \\
\hline $\mathbf{4}$ & 0 & 2 & 0.015625 & 0 & 0 & 0 & 0 & 0 \\
\hline $\mathbf{4}$ & 0 & 2 & 0.015625 & 0 & 0 & 0 & 0 & 0 \\
\hline $\mathbf{4}$ & 0 & 2 & 0.015625 & 0 & 0 & 0 & 0 & 0 \\
\hline $\mathbf{4}$ & 8 & 2 & 0.015625 & 0.125 & 0.125 & 0 & 0 & 4 \\
\hline $\mathbf{4}$ & 4 & 2 & 0.015625 & 0.0625 & 0.0625 & 0 & 0 & 1 \\
\hline $\mathbf{4}$ & 4 & 2 & 0.015625 & 0.0625 & 0.0625 & 0 & 0 & 1 \\
\hline $\mathbf{4}$ & 8 & 2 & 0.015625 & 0.125 & 0.125 & 0 & 0 & 4 \\
\hline $\mathbf{4}$ & 4 & 2 & 0.015625 & 0.0625 & 0.0625 & 0 & 0 & 1 \\
\hline $\mathbf{4}$ & 8 & 2 & 0.015625 & 0.125 & 0.125 & 0 & 0 & 4 \\
\hline
\end{tabular}

Let, $\left[\mathrm{u}^{*}\left(\mathrm{Z}^{\prime} \mathrm{Z}\right)\right]=a,\left[\mathrm{~W}_{4}\right]=b,\left(a \times b^{\prime}\right)=c, \operatorname{norm}(a)=d, \operatorname{norm}(b)=e$

$$
\begin{aligned}
& \phi_{n}(X)=100 \times\left\{\sum_{m=2}^{d}\left[\mathrm{u}^{\prime}\left(\mathrm{Z}^{\prime} \mathrm{Z}\right) \mathrm{w}_{2 \mathrm{~m}}\right]^{2} /\left\|w_{2 m}\right\|^{2}\right\} /\left\|\mathrm{u} *\left(\mathrm{Z}^{\prime} \mathrm{Z}\right)\right\|^{2} \\
& \phi_{n}(X)=100 \times\left\{\left(c^{2} / e^{2}\right)\right\} / d^{2} \\
& \phi_{n}(X)=92.59 \%
\end{aligned}
$$

Same procedure was used to obtain the percentage rotatability for other factors shown in table 2.

Table 2: Result of percentage Rotatability for factor $q=3$ to 11 .

\begin{tabular}{|c|c|c|c|}
\hline No. of factors $(\mathbf{q})$ & No. of Parameters $(\boldsymbol{h})$ & No. of Runs $(\boldsymbol{N})$ & Percentage Rotatability values \\
\hline 3 & 10 & 12 & 92.59 \\
\hline 4 & 15 & 16 & 50.63 \\
\hline 5 & 21 & 24 & 39.12 \\
\hline 6 & 28 & 32 & 44.86 \\
\hline 7 & 36 & 40 & 47.93 \\
\hline 8 & 45 & 56 & 39.50 \\
\hline 9 & 55 & 60 & 36.15 \\
\hline 10 & 66 & 76 & 28.20 \\
\hline 11 & 78 & 96 & 33.01 \\
\hline
\end{tabular}

\section{Results and Discussion}

Rotatability is an important criterion to be considered when selecting a response surface design. The Small Box-Behnken design is rotatable for factor, $q=3$ at 92.59 percent, near rotatable for factors, $q=4,7$ at 50.63 and 47.93 percent respectively and not rotatable for factors, $\mathrm{q}=5,6,8,9,10$ and 11 at $39.12,44.86,39.50,36.15,28.20$ and 33. 01.

\section{Conclusion}

This paper aimed at applying a measure of obtaining percentage rotatability on the Small Box-Behnken designs to determine if the Small Box-behnken designs are rotatable or not and investigated the extent of rotatability in terms of percentage. The factors, $q$, considered range from 3 to 11. In conclusion, results showed that for factors $q$, the Small Box-Behnken design is rotatable for $q=3$ factor, near rotatable for $q=4,7$ factors and not rotatable for $q=5,6,8,9,10$ and 11 factors. However, it is therefore recommended that, where it is possible, $q=3$ factors, should be used in response surface exploration using the Small Box-Behnken design, but where it is not possible to use only $q=3$ factors, the $q=4$ and 7 factors, should be considered since they have more 47 percent rotatability. For future research work, we suggest that a diagnostic check be carried out on Small Box-Behnken design for factors, $q=5,6,8,9,10$ and 11 and recomputed the percentage rotatability. 
Acknowledgement: The effort of Prf. P.E. Chigbu, who supervised my M.Sc. research work that formed the basics of the paper, is acknowledged. We also acknowledge the University of Nigeria Nsukka, where the abstract was published.

\section{References}

[1] Aslan, N. and Cebeci, Y. (2006). Application of Box-Behnken design and response surface methodology for modeling of some Turkish coals. Science Direct, 81, 90 - 97.

[2] Borkowski, J.J. (2003). A comparison of prediction variance criteria for response surface designs.Journal of Quality Technology, 35(1), 70-77.

[3] Box, G.E.P. and Behnken, D.W. (1959). Simplex-sum Designs:A Class of Second Order Rotatable Designs derivable from those of First Order. Institute of Statistics Mimeograph Series, No.232.

[4] Box, G.E. P. and Behnken, D.W. (1960). Some New Three Level Designs for the Study of Quantitative Variables.Technometrics, 2(4), 455 475.

[5] Box, G.E.P. and Hunter, J. S. (1957). Multifactor Experimental Designs for Exploring Response Surfaces .Ann.Math. Statist., 28,195 - 241.

[6] Chigbu, P.E. and Ohaegbulem, U.O. (2011). On the preference of replicating factorial runs to axial runs in restricted second-order designs. Journal of Applied Sciences11(22), 3732-3737.

[7] Draper, N. R. and Guttman, I. (1988). An Index of Rotatability. Technometrics, 32,105-111.

[8] Draper, N.R. and Pukelsheim, F.(1990). Another Look at Rotatability. Technometrics, 30, 195-205.

[9] Ekman, C. (1994).A Note on Rotatability. Research Report: 10, ISSS 0349-0834, Department of Statistics, Goteborg University.

[10] Kiwu-Lawrence, F. C., Kiwu, L. C., Bartholomew, D. C. Obite, C. P. and Akanno F. C. (2021). Evaluation and Comparison of Three Classes of Central Composite Designs. Asian Journal of Probability and Statistics, 13(2): 31-47, 2021; Article no.AJPAS.68613.

[11] Khuri, A.I. (1988). A Measure of Rotatability for Response Surface Designs. Technometrics, 30, 95-104.

[12] Montgomery, D. C. (2005). Design and Analysis of Experiments. 6th ed. John Wiley and Sons Inc, N.J.

[13] Mutai, C., Koske, J. K., and Mutiso, J. M. (2013). A new Method of Constructing Third Order Rotatable Design. Far-East Journal of Theoretical Statistics,42(2), 151 - 157.

[14] Onukogu, I.B. and Chigbu, P.E. (eds.) (2002). Super Convergent Line Series in Optimal Design of Experiment and Mathematical Programming. AP Express Publishers, Nsukka, Nigeria.

[15] Pengpeng, Q., Mingcan, C., Kyoungglim, K., Beomguk, P., Youngyu, S., Eunkyung, K., Min, J., and Jeehyeong, K. (2014). Application of BoxBehnken Design for Modeling and Optimizing Ultrasonic Oxidation of Arsenite with H2O2. Central European Journal of Chemistry, 12(2), 164 $-172$.

[16] University of NigeriaNsukka, M.Sc Research Work, Abstract Publication Link: https://oer.unn.edu.ng/read/on-the-rotatability-property-ofthe-small-box-behnken-design? $r d r=1$

[17] Zhang, T-F, Yang, J-F and Lin, D.K.I. (2011). Small Box-Behnken Design. Statistics and Probability Letter, 81, 1027 - 1033. 